\title{
Electric and photophysical properties of holographic and electroluminescent media based on amorphous molecular semiconductors
}

\author{
A.J. Al-Kadhimi'), N.A. Davidenko, N.A. Derevyanko ${ }^{1)}$, \\ A.A. Ishchenko'), N.G. Kuvshinsky, V.A. Pavlov \\ Kiev Taras Shevchenko National University, 64 Volodymyrska vul., 01033 Kyiv, Ukraine \\ ${ }^{1)}$ Institute of Organic Chemistry, NAS of Ukraine, 5 Murmanska vul., 02094 Kyiv, Ukraine \\ 2) National Technical University "KPI", 37 Peremogy prosp., 03056 Kyiv, Ukraine
}

\begin{abstract}
Results of investigations of electric and photophysical properties inherent to films of amorphous molecular semiconductors doped with different sensitizers of photoconductivity for visible light are discussed. Such films are used in electrography, holography and electroluminescent devices. The general regularities of charge carriers transport, electron-hole pair formation, charge carriers recombination are ascertained. Examples of the holographic recording media as well as media for electroluminescent devices are presented.
\end{abstract}

Keywords: information recording, electroluminescence, dyes, photogeneration, electron-hole pair, recombination, spin conversion.

Paper received 13.06.02; accepted for publication 10.12.02.

\section{Introduction}

Amorphous molecular semiconductors (AMS) are widely used as optical media in electrography and holography $[1,2]$, in photoelectric converters of solar energy [3], in electroluminescent devices [4]. AMS are also used as media with photorefractive properties. Perspectives of practical applications stimulate experimental and theoretical investigations of the features of transport, photogeneration and recombination of charge carriers in these materials. The main results were obtained for the films of poly-N-vnylcarbazole (PVC) and poly-N-epoxypropylcarbazole (PEPC) doped with different sensitizers for visible light $[1,2,5]$. The PVC and PEPC films possess true absorption and photoconductivity for the light wavelength $\lambda<400 \mathrm{~nm}$. In the case of sensitization of these polymers, for example by 2,4,7-trinitro-9-fluorene (TNF), the optical absorption and photoconductivity appear within the visible part of spectrum due to formation of CT-complexes between carbazole and TNF. CT-complexes are the centers of light absorption, photogeneration and recombination of holes $\left(p^{+}\right)$and electrons $\left(e^{-}\right)$. The mechanism of photogeneration consists of two stages. During the first one, after absorption of light quantum in the CT-complexes geminal electron-hole pair (EHP) appears. In the EHP, positive charges $p^{+}$and $e^{-}$are localized at the carbazole group and TNF molecule, respec- tively. Quantum yield of EHP formation in these doped polymers is close to unity. Photoluminescence and recombination luminescence at the room temperature are absent. In the external electric field geminal recombination of $p^{+}$and $e^{-}$in EHP is decreased due to their recombination. At the dissociation of EHP, the charges become separated - the transport of $p^{+}$and $e^{-}$happens through their transitions between carbazole groups and TNF molecules, respectively. This fact means that the carbazole groups and acceptor molecules form the respective transport bands for $p^{+}$and $e^{-}$. The Onsager model is usually used for description of the process of EHP dissociation in dependency on the strength of external electric field $(E)$ and temperature $(T)$ [5]. Analytical expression for this model at the large $E$ coincides with the analytical expression for the probability of charges separation within the scope of the Poole-Frenkel model [2,5]. The probability $(\eta)$ of the EHP dissociation into free current carriers is described by the following dependency:

$\eta \sim R_{n} R_{p} \exp \left(-R_{n} / \alpha_{n}-R_{p} / \alpha_{p}\right) \cdot \exp \left(-\left(W_{0 \mathrm{PH}}-\beta E^{1 / 2}\right) \times\right.$ $\left.\times\left(1 / T-1 / T_{0}\right) / k_{B}\right)$,

where $R_{n}$ and $R_{p}$ are the mean distances between acceptor molecules and carbazole groups, respectively, $\alpha_{n}$ and $\alpha_{p}$ are the radii of localization of electron at the acceptor 
and hole at the carbazole, respectively, $W_{0 \mathrm{PH}}$ is the activation energy of the photogeneration which is equal to the energy of Coulomb interaction between the electron and hole in EHP, $\beta$ and $k_{B}$ are Poole-Frenkel and Boltzmann constants, $T_{0}$ is the characteristic temperature $(490 \pm 15) \mathrm{K}$ for PEPC.

Determined general regularities of electric and photophysical properties of AMS are utilized for development of the recording and electroluminescent media. It turned out that not all sensitizers satisfy the condition of effective photogeneration and radiative recombination of charge carriers. Our viewpoint concerning the problem of sensitizer choice providing the notified requirements is described at the present work.

\section{Model considerations}

Fundamental model conceptions about the photogeneration and transport of charge carriers were achieved at the investigations of photophysical properties of the films of PVC and PEPC with TNF and verified at the investigations of AMS with another sensitizers [6,7]. Similarity of the regularities allows to propose the adequate model of the photogeneration. The respective scheme is presented in Fig.1. Here $\mathrm{P}$ is the efficiency of photoexcitation of molecules from the ground singlet state $\left(\mathrm{S}_{0}\right)$ into the excited (not relaxed) singlet state $\left(\mathrm{S}_{1}{ }^{\mathrm{u}}\right)$ of the photogeneration center; $N_{1}$ and $N_{3}$ are the concentrations of the molecules of photogeneration center in ground relaxed singlet $\left(\mathrm{S}_{1}{ }^{0}\right)$ and triplet $\left(\mathrm{T}_{1}{ }^{0}\right)$ excited states respectively; $n_{1}$ and $n_{3}$ are the EHP concentrations in singlet $(\mathrm{S})$ and triplet states $\left(\mathrm{T}_{0}, \mathrm{~T}_{-}, \mathrm{T}_{+}\right)$respectively $[8,9]$; $k_{U}$ is the constant of internal conversion $\mathrm{S}_{1}{ }^{\mathrm{u}}-\mathrm{S}_{0} ; k_{S U}$ and $k_{S}$ are the velocity constants of non-radiating and radiative transitions $\mathrm{S}_{1}{ }^{0}-\mathrm{S}_{0}$, respectively; $k_{T}$ is the velocity constant of the transition $\mathrm{T}_{1}-\mathrm{S}_{0} ; k_{2}$ and $k_{-2}$ are the velocity constants of intramolecular interconversion $\mathrm{S}_{1}-\mathrm{T}_{1}$ and $\mathrm{T}_{1}-\mathrm{S}_{1} ; k_{1}$ and $k_{-1}$ are the velocity constants of formation and recombination of singlet EHP; $k_{3}$ and $k_{-3}$ are the velocity constants of formation and recombination of triplet EHP; $k_{S T}$ is the velocity constant of spin conversion of EHP; $k_{\eta}$ is the velocity constant of EHP dissociation into free $e^{-}$and $p^{+}$. In a number of cases this scheme is enough for the description of experimentally observed dependencies of $\eta$ on $E, T, \lambda$ and on the structure and concentration of molecules of photogeneration centers as well as molecules forming the respective transport bands

\section{Results}

Since usually $k_{-1}>k_{-3}$, from the scheme presented in Fig. 1 follows that $\eta$ increases when the ratio $n_{3} / n_{1}$ increases under conditions:

$k_{2}>k_{-2} ; k_{1}>k_{S} ; k_{3}>k_{T} ; k_{\eta}>k_{-1}, k_{-3}$

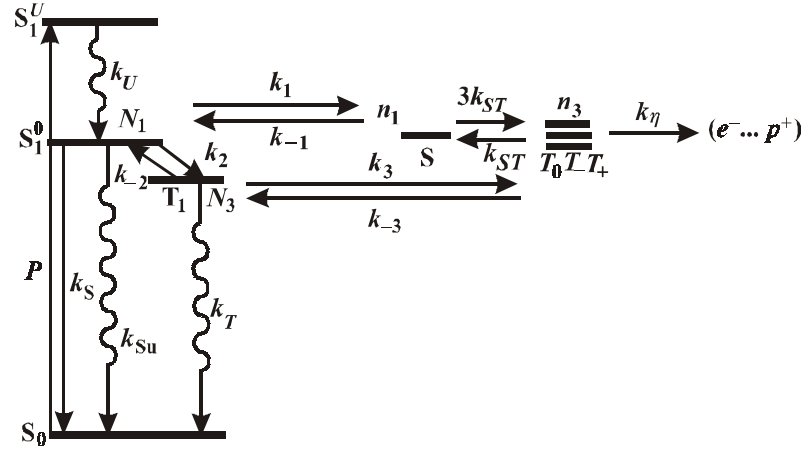

Fig. 1. The scheme of electronic processes during the photogeneration and recombination of charges.

It could be observed for example: i) under growth of the structural rigidity of the molecules [10], when surplus of the vibration energy in the process of internal conversion $\mathrm{S}_{1}{ }^{\mathrm{u}}-\mathrm{S}_{0}$ involves the strengthening of the interconversion; ii) in physical dimers and aggregates of dyes [11,12], when sufficiently large overlapping of S- and T-states exists; iii) when using magnetically active ions of ionic dyes [13], when undyed gegenion inflies on the S-T-conversion of EHP. It should be pointed out that in large external electric field a possibility of thermo-field ionization of the photogeneration centers from the excited states with short lifetime is non-excluded. $k_{1}$ and $h$ increase under these conditions. Influence of the structural rigidity of the polymer and dopants on the dissociation and recombination of charge carriers was taken into account in the proposed scheme. Velocity constant $k_{S T}$ depends on energy dissipation of electron and hole. Therefore, velocity of transfer of the thermal energy from the photogeneration center and $k_{S T}$ decrease with growing structural rigidity of the molecules where charge carriers in EHP are localized [10]. Thus, electric and photophysical properties of AMS can be controlled not only by change of their $p$-electronic system, but also by variation of structural rigidity of polymer and dopants.

The photogeneration of triplet EHP with higher lifetimes as compared to those of singlet EHP should be considered as an essential factor. The conclusion about the influence of velocities of the spin conversion on the efficiency of photogeneration, recombination and dissociation of EHP is based on observed effect of magnetic field on photoconductivity and photoluminescence [8,9] in AMS with organic acceptors, with compounds characterized by an intramolecular charge transfer and with dyes. For express-analysis of the ratio between $n_{1}$ and $n_{3}$ as well as for analysis of the efficiency of the photogeneration of $p^{+}$and $e^{-}$, the method to estimate photoconductivity changes during interaction of EHP with triplet excitons $[9,13]$ could be employed. In this case, $k_{S T}$ increases at interaction of EHP with molecular excitons. Growth of $k_{S T}$ can be revealed also under decrease of light wavelength exciting the photogeneration centers. When cationic dyes are used as the photogeneration centers the strengthening of the respective quan- 


\section{A.J. Al-Kadhimi et al.: Electric and photophysical properties of holographic...}

tum yield of their photoluminescence is observed within the short-wave part of the absorption spectrum dye due to contribution of recombination luminescence [14]. Thus, organic dyes serve as centers of photogeneration and radiative recombination of non-equilibrium charge carriers [15] in AMS. This is an important aspect for development of new photoconducting and electroluminescent materials.

\section{Media for electrography, holography and electroluminescent devices}

Results of investigations of electric and photophysical properties of AMS were used for our developments of new holographic media for devices of holographic interferometry, where the thermoplastic method of holographic recording is used [2]. High results demonstrate the memory medium for thermoplastic recording based on films PEPC+1mass. \%HISD, where squarilic dye HISD is used [16]:

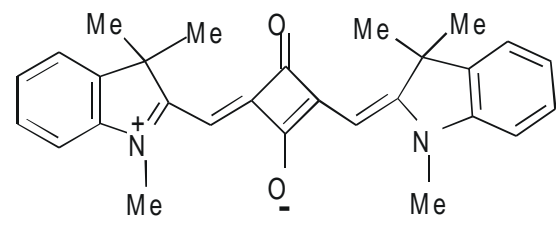

Maximum of optical absorption of the films PEPC +1 mass. $\%$ HISD is close to light wavelength $\lambda=$ $=655 \mathrm{~nm}$. For the laser with $\lambda=633 \mathrm{~nm}$ the values of diffraction efficiency are $23-25 \%$, and holographic sensitivity $20-30 \mathrm{~m}^{2} / \mathrm{J}$ were achieved for the ratio between intensities of reference and object beams 1:1. The band of optimal spatial frequencies of transmissive characteristic is $20-1500 \mathrm{~mm}^{-1}$, the optimal spatial frequency range is $350-1000 \mathrm{~mm}^{-1}$. The basic information characteristics did not become worse after many cycles of recording and erasing the holograms (more than 600 cycles). The signal-to-noise ratio at holographic readout was not lower that 100 . Such results are provided by high efficiency of photogeneration of charge carriers from the molecules of intraionic dyes HISD. The latter factor is caused by dominant formation of triplet EHP during photogeneration [12], going out of the holes as well as electrons from HISD is possible. The latter circumstance, in accordance to (1), involves considerable growth of the probability of EHP dissociation due to reduction of the probability of charges recombination.

Investigations of the features of charges recombination is important for development of new materials for the electroluminescent devices. This process is competitive respectively to appearance of free charge carriers. Moreover, if ratiative channel of degradation of excitation energy of the molecules is ruinous for the processes of charge carriers photogeneration, then this channel is the most effective for acceleration of the charge pair recombination. That is why, in accordance to the scheme in Fig. 1 realization of the conditions opposite to (2) is desirable for the effective recombination. It should be specially pointed out that, for the effective radiative recombination conditions, $k_{-1}>k_{-3}$ and $k_{S T}>k_{\eta}$ are the most important. The latter means that, during charge recombination in the contact EHP, ratio between singlet and triplet states can increase and maximal probability of the radiative recombination can reach essentially larger value than $1 / 4$.

We have used the results of investigations of the features of charge carriers recombination for development of electroluminescent media based on AMS films. It turned out that employing of boron-fluoride complexes is the most effective, for example BFI [16]:

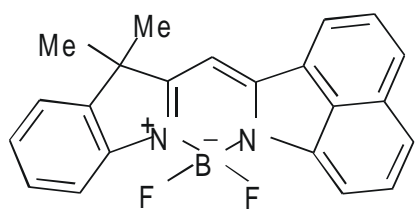

Growth of BFI concentration (N) in the samples $\mathrm{SnO}_{2}: \mathrm{In}_{2} \mathrm{O}_{3}$-PEPC+Nmass. $\% \mathrm{BFI}-\mathrm{Al}$ involves appearance of the electroluminescence. Under $\mathrm{N}$ growth from 1 till 50 vibrating structure of the spectra of optical absorption becomes more smooth and spectra are widened. The maximum of the absorption band for the films PEPC + Nmass. $\%$ BFI shifts from $\lambda=530 \mathrm{~nm}$ to $\lambda=510$ $\mathrm{nm}$, the fluorescence maximum changes from $\lambda=612 \mathrm{~nm}$ to $\lambda=638 \mathrm{~nm}$. Such changes in the spectra is indicative [16] of appearance of intermolecular interactions in the system dye-dye. The limiting state of these interaction is aggregation. The spectra of photoluminescence and electroluminescence practically coincide. The most bright electroluminescence is demonstrated by the samples with $N=50$. The brightness is close to that for electroluminescence in similarly prepared samples $\mathrm{SnO}_{2}: \mathrm{In}_{2} \mathrm{O}_{3}-\mathrm{PPV}-\mathrm{Al}$, where PPV is the poly(p-phenylenevinylenes) (commercial) widely used in the electroluminescent devices [4].

The peculiarities of electroluminescence in the samples with films PEPC+Nmas.\%BFI could be explained in the following manner. The holes and electrons appear in the polymer film due to thermofield injection from the electrodes and drift in the external electric field. If the carrier concentration is large, then their meeting at the distances comparable both with the Onsager radius [5] and spin correlation radius [8] or less is very probable. The initial non-correlated spin state of electron and hole with probability $3 / 4$ and $1 / 4$ for triplet and singlet states, respectively [17], can change. As the result of spin conversion singlet pair of opposite charges can arise from initially triplet EHP: anion-radical $\mathrm{BFI}^{-}{ }^{-}$and cationradical $\mathrm{PEPC}^{+}{ }^{+}$. At the aggregation of dye the difference between its LUMO and HOMO as well as between HOMO and the Fermi level of the cathode metal decreases. The latter fact contributes to lowering the energetic barrier for thermofield injection of electrons from cathode into the AMS volume. Besides, collectivization of orbitals of dye molecules in the aggregated state facilitates transitions of injected electrons during their 


\section{A.J. Al-Kadhimi et al.: Electric and photophysical properties of holographic...}

transport. The efficiency of spin conversion is caused by the presence of the coordinated boron atom in the BFI molecule. Spin density of unpaired electron and the largest negative charge in the $\mathrm{BFI}^{--}$anion-radical are localized exactly close to this atom. Such anion-radical belonging to contact EHP contributes to growth of the probability of charge recombination due to some reasons. First of all, point charge possesses larger energy of electrostatic interaction with the charge of opposite sign at small distances as compared to the delocalized one [18]. That is why charged boron atom in the anion-radical BFIinvolves capture and holding of the drifting hole. Secondly, the boron atom consists of enough magnetically active nucleus $\mathrm{B}^{11}$ (magnetic momentum $\mu=2.6885$ ). Besides, boron atom belonging to the molecule BFI is coordinated by two fluorine atoms consisting of also magnetically active nucleus $\mathrm{F}^{19}(\mu=2.6288)$. Therefore, changes of the non-correlated spin state of the ion-radical pair happen due to hyperfine interaction with the boron and fluorine nucleuses during the time interval when the hole is held close to the charged and coordinated boron atom. This is the basic reason of effective spin conversion.

\section{Conclusions}

Thus, the choice of amorphous molecular semiconductors is determined by the technical problems of their usage. The type and concentration of photogeneration centers of current carriers should by chosen in proper way to reach the high quantum yield of EHP photogeneration, small probability of EHP recombination and capturing the carriers by traps, high velocity of electrons and holes drift, low electroconductivity. Such choice allows to achieve high photoconductivity of the electrographic and holographic recording media. To reach high brightness of radiation in electroluminescent media based on polymer compositions the type and concentration of dopants should provide the high efficiency of injection of positive and negative charge carriers from electric contacts into the volume of polymer film, high velocity of electrons and holes drift till their meeting, high probability of meeting of opposite charge carriers, high velocity of spin conversion in contact EHP for their transition from the state with non-correlated spins into the singlet spin state from which one singlet excited state of recombination center arises.

\section{References}

1. P.M. Borsenberger, D.S. Weiss. Photoreceptors for Xerography. Marcel Dekker, New York, (1998).

2. N.G. Kuvshinsky, N.A. Davidenko, V.M. Komko. Physics of Amorphous Molecular Semiconductors. Lybid', Kiev, 1994.

3. C. Murray, D. Norris, M. Bawendy // J. Am. Chem. Soc., 115, pp.8706-8711 (1993).

4. U. Mitschke, P. Bauerle // J. Mater. Chem., 10(8), pp.14711478 (2000).

5. M. Pope, C.E. Swenberg. Electronic Processes in Organic Crystals and Polymers. $2^{\text {nd }}$ ed. University Press, Oxford, (1999).

6. N.A. Davidenko, N.G. Kuvshinsky. // J. Inf. Rec. Mats., 21, pp.185-191 (1993).

7. N.A. Davidenko, N.G. Kuvshinsky, V.G. Syromyatnikov, L.N. Fedorova. // Advanced Materials for Optics and Electronics,. 7, pp.207-211 (1997).

8. N.A. Davidenko, N.G. Kuvshinsky. // J. Chem. Phys., 106(15), pp.6507-6511 (1997).

9. N.A. Davidenko, N.G. Kuvshinsky. // Advanced Materials for Optics and Electronics, 7, pp.255-259 (1997).

10. N.A. Davidenko, N.A. Derevyanko, A.A. Ishchenko, V.A Pavlov, S.L. Studzinsky. // Theor. and Exper. Chem., 37, pp.241-246 (2001).

11. N.A. Davidenko, A.A. Ishchenko, I.L. Mushkalo, V.A. Pavlov. // Theor. and Exper. Chem., 34(6), pp.371-376 (1998).

12. N.A. Davidenko, A.A. Ishchenko, V.A. Pavlov. // Sci. Appl. Photo., 41(2), pp.169-174 (1999).

13. N.A. Davidenko, A.A. Ishchenko.// Chem. Phys., 247, pp.237-243 (1999)

14. N.A. Davidenko, N.A. Derevyanko, A.A. Ishchenko, N.G. Kuvshinsky, V.G. Syromyatnikov, S.L. Studzinsky, L.N. Fedorova // Journal of Applied Spectroscopy, 68, pp.450455 (2001).

15. N. Davidenko, A. Ishchenko. // Advanced Materials for Optics and Electronics, 8, pp.201-207 (1998).

16. A.A. Ishchenko. Structure and Spectral Luminescence Properties of Polymethine Dyes. Naukova Dumka, Kiev, (1994).

17. A.L. Buchachenko, E.L. Frankevich. Chemical Generation and Reception of Radio- and Microwaves, $\mathrm{VCH}$ Publishers, New York, (1993)

18. N.G. Kuvshinsky, N.A. Davidenko, V.V. Reshetnyak, L.I. Savransky, V.L. Sheptun. // Chem. Phys. Lett., 165(4), pp.323328 (1990). 